\title{
Intracellular Sphingosine-1-Phosphate Receptor 3 Contributes to Lung Tumor Cell Proliferation
}

\author{
Michela Terlizzia Chiara Colarusso ${ }^{a} \quad$ Giusy Ferraro $^{a} \quad$ Maria Chiara Montia \\ Ilaria De Rosa ${ }^{b}$ Pasquale Somma ${ }^{b}$ Rosario Salvic Aldo Pinto ${ }^{a}$ \\ Rosalinda Sorrentino ${ }^{a}$ \\ aUniversity of Salerno, Department of Pharmacy, DIFARMA, Fisciano, Italy, bAnatomy and Pathology \\ Unit, Ospedale dei Colli, AORN, "Monaldi", Naples, Italy, 'Thoracic Surgery Unit, Ospedale dei Colli, \\ AORN, "Monaldi", Naples, Italy
}

\section{Key Words}

Sphingosine-1-phosphate $(\mathrm{S} 1 \mathrm{P}) \cdot \mathrm{S} 1 \mathrm{P}$ receptors $\cdot \mathrm{S} 1 \mathrm{PR} 3 \cdot$ Smoking $\bullet$ Lung cancer

\begin{abstract}
Background/Aims: The pleiotropic lipid mediator sphingosine-1-phosphate (S1P) exerts a multitude of effects on respiratory cell physiology and pathology through five S1P receptors (S1PR1-5). Epidemiological studies proved high levels of circulating S1P in non-small cell lung cancer (NSCLC) patients. Studies in literature suggest that high levels of S1P support carcinogenesis but the exact mechanism is still elusive. The aim of this study was to understand the mechanism/s underlying S1P-mediated lung tumor cell proliferation. Methods: We used human samples of NSCLC, a mouse model of first-hand smoking and of Benzo(a)pyrene (BaP)induced tumor-bearing mice and A549 lung adenocarcinoma cells. Results: We found that the expression of S1PR3 was also into the nucleus of lung cells in vitro, data that were confirmed in lung tissues of NSCLC patients, smoking and tumor bearing BaP-exposed mice. The intranuclear, but not the membrane, localization of S1PR3 was associated to S1P-mediated proliferation of lung adenocarcinoma cells. Indeed, the inhibition of the membrane S1PR3 did not alter tumor cell proliferation after Toll Like Receptor (TLR) 9 activation. Instead, according to the nuclear localization of sphingosine kinase (SPHK) II, the inhibition of the kinase completely blocked the endogenous S1P-induced tumor cell proliferation. Conclusion: These results prove that the nuclear S1PR3/SPHK II axis is involved in lung tumor cell proliferation, highlighting a novel molecular mechanism which could provide differential therapeutic approaches especially in
\end{abstract} non-responsive lung cancer patients. 


\section{Introduction}

Recent evidence has highlighted the relevance of the axis sphingosine/sphingosine-1phosphate (S1P) in both physiological and pathological conditions [1-4]. Sphingosine can derive from both the activation of sphingomyelinase and/or serine-palmitoyltransferase [5]. The sphingomyelinase is an enzyme which induces membrane sphingolipids degradation to generate ceramide, which in turn is converted by the ceramidase into sphingosine. The alternative pathway for sphingosine synthesis is related to the serine-palmitoyltransferase which promotes the so-called de novo synthesis through the condensation of serine to palmitoyl-CoA [6]. Both metabolic pathways lead to ceramide generation that on one side could be transformed into ceramide-1-phosphate (C1P) and, on the other lead to the formation of sphingosine, which will be phosphorylated by sphingosine kinases I and/or II (SPHK I and/or SPHK II). Once S1P is generated, it is transported through the cell membrane in an inside-out mode by means of Spinster homolog 2 (SPNS2), a non-ATP-dependent organic ion transporter [7], so that it can interact in a paracrine and/or autocrine manner with its receptors, sphingosine-1-phosphate receptors (S1PRs). So far, five G protein-coupled S1PRs have been identified, S1PR1, S1PR2, S1PR3, S1PR4 and S1PR5, which are involved in physiological and pathological conditions, including cell growth, apoptosis, trafficking, differentiation and activation of immune cells, inflammation, cellular architecture and vascular integrity regulation [8-11].

So far, S1PR1, a Gi-coupled receptor, and S1PR3, a Gi/q-coupled receptor, have been demonstrated as involved in tumor growth. In particular, S1PR1 is largely considered as a pro-tumorigenic receptor in that it promotes neo-angiogenesis [12], metastasis of various cancers (i.e., breast and colorectal cancer, thyroid carcinoma), via the activation of STAT3, extracellular signal-regulated kinase (ERK), Akt, and Rac pathways [13]. Similarly, S1P/ S1PR3 axis is associated with proliferation, migration and angiogenesis of cancer cells [14] via the activation of TGF- $\beta$ /SMAD3 axis [15]. Little is known about S1PR2 and S1PR4, although it looks like that their activity is majorly on immune cells $[16,17]$.

In our recent study we found that S1P supported Toll-like Receptor (TLR)-9/NF- $\kappa B-$ mediated TNF- $\alpha$ release from lung adenocarcinoma cells, but not from non-pathological cells, mainly via the inside-out transport of TLR-9-induced S1P which interacted with S1PR3 [18]. In support, epidemiological studies have revealed that higher levels of S1P are detected in patients with chronic respiratory disorders, but in particular, in lung cancer patients [19, 20].

Based on our previous study in which we found that S1P/S1PR3 is involved in an inflammatory pattern via the activation of TLR-9, highly expressed on lung cancer cells, the main goal of the current study was to better understand the involvement of S1P/ S1PR3 pathway/signaling during lung carcinogenesis, taking advantage of a mouse model of first-hand smoke exposure and of a carcinogen-induced lung cancer. We found that the intranuclear, but not the membrane, localization of S1PR3 was associated to the proliferation of lung adenocarcinoma cells, mechanism that was correlated to human and mouse samples of smoke-exposure and carcinogen-induced lung cancer, which were characterized by higher utilization of S1P.

\section{Materials and Methods}

\section{Human tissues}

Samples human tissues were obtained by patients with diagnosis of Non-Small Cell Lung Cancer (NSCLC) or non-neoplastic pulmonary pathologies (i.e tuberculosis, emphysema) who underwent surgical resection at Ospedale dei Colli, AORN, Monaldi, Naples, Italy, during the period 2014-2017. Clinical data, lifestyle and histology reports were obtained from the Pathological Anatomy Unit of the hospital. The project was approved by the institutional review board and by the Ethical Committee (approval number for lung cancer patients 1254/2014). Samples from lung cancer and non-cancerous patients were collected 


\section{Cellular Physiology Cell Physiol Biochem 2021;55:539-552 \\ \begin{tabular}{l|l|l} 
and Bincl $10.33594 / 000000431$ & 2021 The Author(s). Published by
\end{tabular} \\ \begin{tabular}{l|l} 
Published online: 3 September 2021 & Cell Physiol Biochem Press GmbH\&Co. KG \\
\hline
\end{tabular} \\ Terlizzi et al.: Intracellular Activity of S1P in Lung Tumor Cells}

after oral and written information provided by the MDs, and after the signature of a consent form before entering the project. Samples were collected and used within 24 hours. Lung cancer patients were $60 \pm 10$ (mean \pm S.E.M.) years of age.

\section{Mice}

Female specific pathogen-free C57Bl/6N mice (6-8 weeks of age) (Charles River Laboratories, Lecco, Italy) were fed with a standard chow diet and maintained in specific pathogen-free conditions at the animal care facility of Department of Pharmacy, University of Salerno. This study was carried out in strict accordance with the recommendations in the Guide for Care and Use of Laboratory Animals of the Health National Institute. The experimental protocol was approved by the Ethical Committee for Animal Studies of the University of Salerno and Health Ministry with the approval number 985/2017. All animal experiments were performed under protocols that followed the Italian (D.L. 26/2014) and European Community Council for Animal Care (2010/63/EU). Experiments were repeated twice. Each group was composed of 9 mice.

Cigarette smoke mice-exposure protocol

As previously reported [21], mice were exposed to first-hand smoking by means of a nose-only chamber (EMMS, UK). Briefly, mice were exposed to first-hand smoking once a day at the concentration of $1 \mu \mathrm{g} / \mathrm{cm}^{3}$ of total particulate matter (TPM), generated from Red Marlboro cigarettes (TAR = $12 \mathrm{mg} / \mathrm{cig}$ ), 5 days/week for 8 and 16 weeks. Control mice, here defined as Room Air group, breathed filtered air for the same time. Mice were sacrificed 24 hours after the last cigarette smoke (CS) exposure.

\section{Carcinogen-induced lung cancer in mice}

Mice were anesthetized with isoflurane and intratracheally (i.t.) instilled with Benzo(a)pyrene (BaP) (SUPELCO Analytical, 595 North Harrison Road, Bellefonte, PA, USA; Cat\#40071) at the dose of $1 \mu \mathrm{g} / \mathrm{mouse}$ in $10 \mu \mathrm{l}$ at week 1, 2, 3, week 8-9-10 and week 12-13-14. Vehicle group (acetone 0.1\%; Sigma-Aldrich, 3050 Spruce Street, St. Louis, MO, USA; Cat\#32201) was considered. Lungs were isolated and left lung lobes were embedded into OCT medium to perform H\&E staining as described below. Right lung lobes were digested with 1U/mL collagenase (Sigma Aldrich, 3050 Spruce Street, St. Louis, MO, USA; Cat\#C0130). Cell suspensions were passed through $70 \mu \mathrm{m}$ cell strainers, and red blood cells were lysed. Cell suspensions were used for flow cytometric analyses of different cell subtypes. Lungs were homogenized and western blotting were performed. This study was carried out in strict accordance with the recommendations in the Guide for the Care and Use of Laboratory Animals of the National Institutes of Health. The protocol was approved by the Committee on the Ethics of Animal Experiments of the University of Salerno and by National Institutes of Health with the approval number 683/2019.

Tumor lesion evaluation

H\&E staining was performed to evaluate tumor lesion development after carcinogen exposure. Tumor lesion was expressed as ratio of Tumor Area/Total Lung Area, calculated with ImageJ software (NIH, USA).

\section{Flow Cytometry Analysis}

Cell suspensions obtained by collagenase digested lungs were analysed using flow cytometry (BD FacsCalibur, Milan, Italy) to evaluate the infiltration and the nature of immune cells recruited to the lung of mice. Cell suspensions were labelled with specific antibodies (CD11b: 8999 BioLegend Way, San Diego, CA; Cat\#101239; Gr-1: 8999 BioLegend Way, San Diego, CA; Cat\#108408; Arginase-1: Proteintech, 5400 Pearl Street, Rosemont, IL, USA; Cat\#16001-1-AP; CD3: 8999 BioLegend Way, San Diego, CA; Cat\#100322; CD4: 8999 BioLegend Way, San Diego, CA; Cat\#130308; CD25: 8999 BioLegend Way, San Diego, CA; Cat\#101904; FoxP3: Thermo Fisher Scientific, 3747 Meridian Road, Rockford, IL, USA; Cat\#PA5-12396).

\section{Western blotting analysis}

Human and mouse lung homogenates were used to examine the expression of S1PR1 (Santa Cruz Biotechnologies, Heidelberg, Germany; Cat\#sc-48356), S1PR3 (Alomone labs, Jerusalem, Israel; Cat\#ASR-013), SPNS2 (Abcam, Milan, Italy; Cat\#ab82629), ASAH1 (N-acylsphingosine amidohydrolase 1, ASAH1 or ceramidase; Elabscience; US; Cat\#E-AB-10959). GAPDH (OriGene Technologies, Rockville, MD; Cat\#TA890003) and HSC70 (OriGene Technologies, Rockville, MD; Cat\#TA332519) were used as loading protein. Data were analysed by means of ImageJ software (NIH, USA). 


\section{Cellular Physiology Cell Physiol Biochem 2021;55:539-552 \begin{tabular}{ll|l}
\cline { 2 - 2 } DOl: 10.33594/000000431 & (c) 2021 The Author(s). Published by
\end{tabular} and BiOChemistry Published online: 3 September 2021 Cell Physiol Biochem Press GmbH\&Co. KG \\ Terlizzi et al.: Intracellular Activity of S1P in Lung Tumor Cells}

\section{Immunofluorescence analyses}

Mice and human lung cryosections were analysed for the presence of FITC-conjugated S1PR3. DAPI (4',6-diamidino-2-phenylindole) was used to stain cell nuclei. Images were observed by means of Carl Zeiss confocal microscopy (magnification: 40X).

\section{Sphingolipids extraction from mouse tissues}

Extraction of sphingolipids, sphingosine (S) and sphingosine-1-phosphate (S1P), from lung homogenates was performed by the following method [22]. Briefly, $1 \mathrm{ml}$ of mixture $i$-PrOH-Water-EtOAc (30:10:60) was added to $0.2 \mathrm{mg}$ of total proteins in each lung tissue homogenate. Samples were stirred for 30 minutes, sonicated for 15 minutes and centrifuged at $21000 \mathrm{~g}$ for 15 minutes. The supernatant was transferred to a new centrifuge tube and evaporated to dryness at $40^{\circ} \mathrm{C}$ in the vacuum rotator. Samples were reconstituted in $50 \mu \mathrm{l} \mathrm{MeOH}$, vortexed for 5 minutes, sonicated for 10 minutes and centrifuged for 10 minutes at $10000 \mathrm{~g}$ and $8 \mu \mathrm{l}$ were loaded on the UPLC-MSMS system.

\section{LC-MS/MS analysis}

Sphingosine and S1P were quantified by liquid chromatography coupled to tandem mass spectrometry detection (LC-MS/MS) on Shimadzu LC-20A and Auto Sampler systems and QTRAP 6500 instrument from AB-Sciex. C18 chromatographic column (Kinetex C18, $50 \times 2.1 \mathrm{~mm}, 5 \mu \mathrm{m}$, Phenomenex) was used for chromatographic separation. The mobile phase was composed of water $0.1 \%$ formic acid (mobile phase A) and methanol $0.1 \%$ formic acid (mobile phase B). The flow rate was set at $400 \mu \mathrm{l} /$ minute. The mass spectrometer was set in the positive ion mode (ESI+) with an electrospray voltage of $5500 \mathrm{~V}$ at $400^{\circ} \mathrm{C}$ of the heated capillary temperature. The multiple reaction monitoring (MRM) mode and the Analyst 1.6.2 software has been used. Sphingosine was analyzed with the mass transition $300 \mathrm{~m} / \mathrm{z}$ to $282 \mathrm{~m} / \mathrm{z}$ and it was observed at the rt of 6.65 minutes. S1P was analyzed with the mass transition $380 \mathrm{~m} / \mathrm{z}$ to $264 \mathrm{~m} / \mathrm{z}$ and it was observed at the rt of 7.95 minutes. Nitrogen was used as the air curtain gas (20psi), atomizing gas (30psi), auxiliary gas (60psi) and collision gas (4psi). Dwell time was $100 \mathrm{~ms}$, DP was 74v, EP was 10v, CE was $22 \mathrm{v}$ and CXP was $15 \mathrm{v}$. For quantitative analysis, a standard curve with sphingosine and S1P amounts of 250-2.5 pg has been run. Data were expressed as sphingosine and S1P Peak Area.

\section{Proliferation assay}

A549 cells (ATCC $® C C L-185^{\mathrm{TM}}$ ) were stained by means of carboxyfluorescein diacetate succinimidylester (CFSE; $1 \mu \mathrm{M}$; proliferation Dye, eBioscience Inc., San Diego, CA; Cat\#65-0850-85) to perform proliferation assay. CFSE flow cytometry data was analysed by means of ModFit4.0 software (BD Pharmingen). A549 cells were treated for 48 hours with sphingosine-1-phosphate (S1P $10 \mathrm{nM}$; Sigma-Aldrich, Merck Life Science S.r.l., Milan, Italy; Cat\#S9666) or unmethylated Cytosine phosphate Guanosine-oligodeoxynucleotides, CpGODN (CpG $1 \mu \mathrm{g} / \mathrm{ml}$; Vincibiochem, Milan, Italy) in presence of specific pharmacological inhibitors, such as TY52156, a S1PR3 receptor antagonist (S1PR3a $10 \mu \mathrm{M}$; Tocris Bioscience, Ellisville, MO; Cat\#5328), antiSpinster homolog 2 antibody ( $\alpha$ SPNS2 $10 \mathrm{ng} / \mathrm{ml}$; Abcam, Milan, Italy; Cat\#ab82629), SKI II, a selective inhibitor of sphingosine kinases (SKI II $10 \mu \mathrm{M}$; Tocris Bioscience, Ellisville, MO; Cat\#2097).

\section{Nuclear and cytosolic protein extraction}

Nuclear and cytosolic protein were isolated from A549 cells, after 3 hours treatment with CpG $1 \mu \mathrm{g} /$ $\mathrm{ml}$, by using Qproteome Nuclear Protein Kit and Qproteome Plasma Membrane Protein Kit according to the manufacturer's instructions (QIAGEN, United Kingdom). S1PR3 and SPHK II expression was evaluated in the different cellular compartments by means western blotting analysis. GAPDH was used as cytosolic loading protein and Lamin A (Sigma-Aldrich, Merck Life Science S.r.l., Milan, Italy; Cat\#L1293) as nuclear loading protein.

\section{Statistical Analysis}

Data are reported as median \pm percentile range and represented as dot plots. Statistical differences were assessed with TWO-WAY or ONE-WAY Analysis of variance (ANOVA) followed by multiple comparison post-tests as appropriate. $p$ values less than 0.05 were considered significant. The statistical analysis was performed by using GraphPad prism 9.0.0 version (San Diego, USA). 


\section{Results}

Sphingosine-dependent pathway in human samples of NSCLC

In our previous study we found that the activation of sphingosine/S1P axis in human tumor cells was associated to the release of a pro-inflammatory cytokine, TNF- $\alpha$ [18]. This effect was not observed in non-cancerous cells (i.e., healthy human fibroblasts), implying that this mechanism was effective in the sole tumor cells. In order to understand whether this pathway was involved in lung carcinogenesis, we used human tissues of NSCLC and non-cancerous patients undergoing thoracic surgery. We found that S1PR3 was expressed in all samples of NSCLC (Fig. 1A). Similarly, it was also detectable in non-cancerous lung samples (Fig. 1A), although at a different content. However, the quantitative analysis performed by ImageJ software did not show statistically differences of S1PR3 expression in cancerous (LK) vs noncancerous lung tissue homogenates (Fig. $1 \mathrm{~A}$ and $1 \mathrm{~B}$ ). Instead, we were not able to detect S1PR1 (data not shown).

The expression of the inside-out S1P transporter, SPNS2, was not altered in tumor tissues (LK) compared to noncancerous tissues (Fig. 1A and 1C). Moreover, we did not find any statistical difference in both non-cancerous and tumor samples in the expression of ceramidase (ASAH1), the enzyme involved in sphingosine synthesis (Fig. $1 \mathrm{~A}, 1 \mathrm{D}$ and $1 \mathrm{E}$ ). However, we have to point out the active form of the ceramidase (ASAH1, 35kDa) was evident in 2 cases of tuberculosis (patient 16 and 36) and in a patient (lane \#84) who had fibrosis (Fig. $1 \mathrm{~A}, 1 \mathrm{E})$.

These data may suggest that sphingosine-dependent signaling is similar in lung tumor cells as well as in non-cancerous cells.

S1P pathway in lung samples of smoking mice

Smoking represents nowadays the high-risk factor that can induce lung cancer. Therefore, we used a model of firsthand smoking mice to evaluate whether smoking-associated lung inflammation, was correlated to sphingosine-dependent pathway. We used lung homogenates of mice which were sacrificed at 8 and 16 weeks, time points that revealed altered

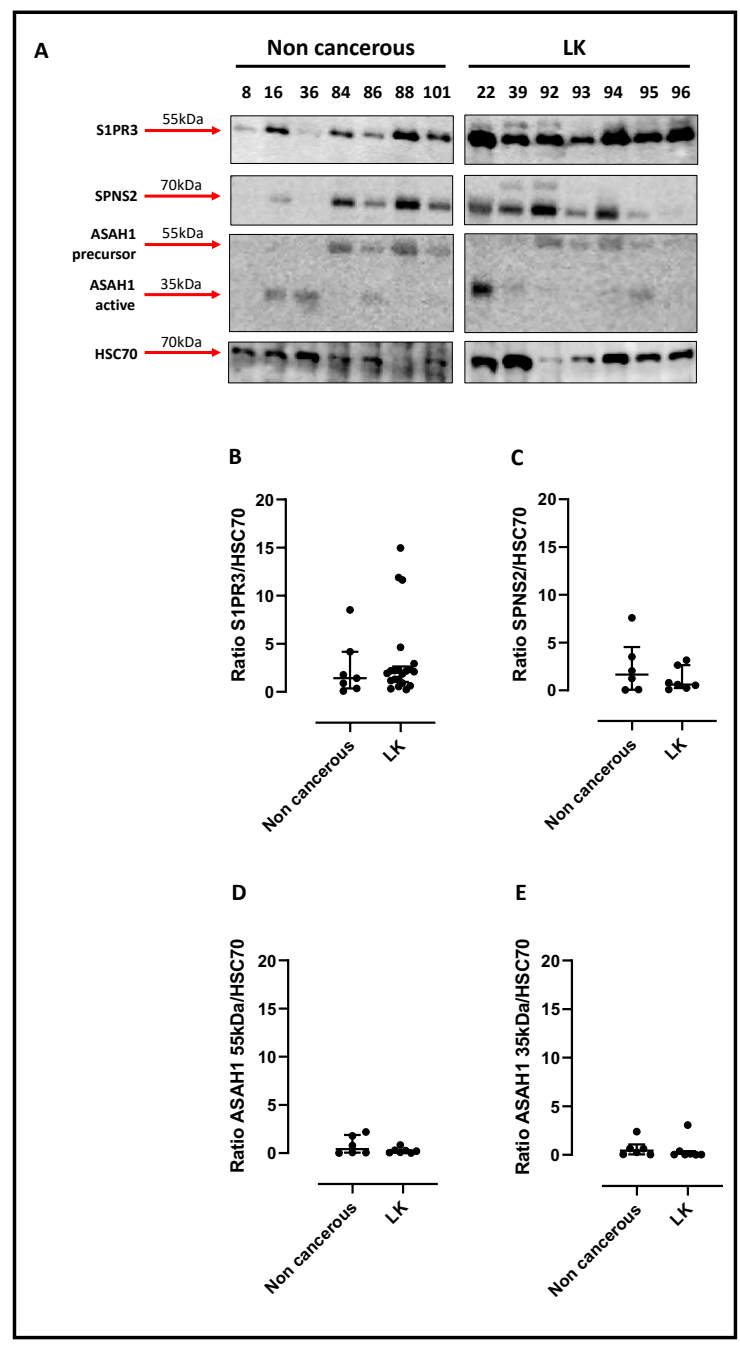

Fig. 1. Western blotting analysis on human lung tissues. S1PR3 (55kDa) (A and B), SPNS2 (70kDa) (A and C) and ASAH1 (precursor 55kDa; active form $35 \mathrm{kDa}$ ) (A, $\mathrm{D}$ and $\mathrm{E}$ ) expression was evaluated on non-cancerous vs cancerous (LK) human lung tissues. HSC70 was used as loading protein. Densitometric analysis is shown. The quantitative analysis was performed by means of ImageJ software. No statistically differences were observed for S1PR3, SPNS2 and ASAH1 expression in cancerous (LK) vs non-cancerous lung tissue homogenates. Data are presented as dot plot, showing the median \pm interquartile range. S1PR3: sphingosine-1-phosphate receptor 3; SPNS2: sphingolipid transporter 2; ASAH1: N-Acylsphingosine Amidohydrolase 1. 
Fig. 2. Western blotting analysis on smoking mice lung tissues. S1PR3 $(55 \mathrm{kDa})$ expression is increased in the lung of smoking mice at 16 weeks compared to 8 weeks and Room Air group (A). ASAH1 active form $(15 \mathrm{kDa})$ is increased in the lung of smoking mice at 16 weeks compared to Room Air group (B). SPNS2 (70kDa) expression (C) is reduced in smoking mice at 16 weeks compared to Room Air. HSC70 was used as loading protein. Densitometric analysis is shown. The quantitative analysis was performed by ImageJ software. Data are presented as dot plot, showing the median \pm interquartile range. Statistical differences were performed by using Mann-Whitney t test. S1PR3: sphingosine-1-phosphate receptor 3; SPNS2: sphingolipid transporter 2; ASAH1: N-Acylsphingosine Amidohydrolase 1.

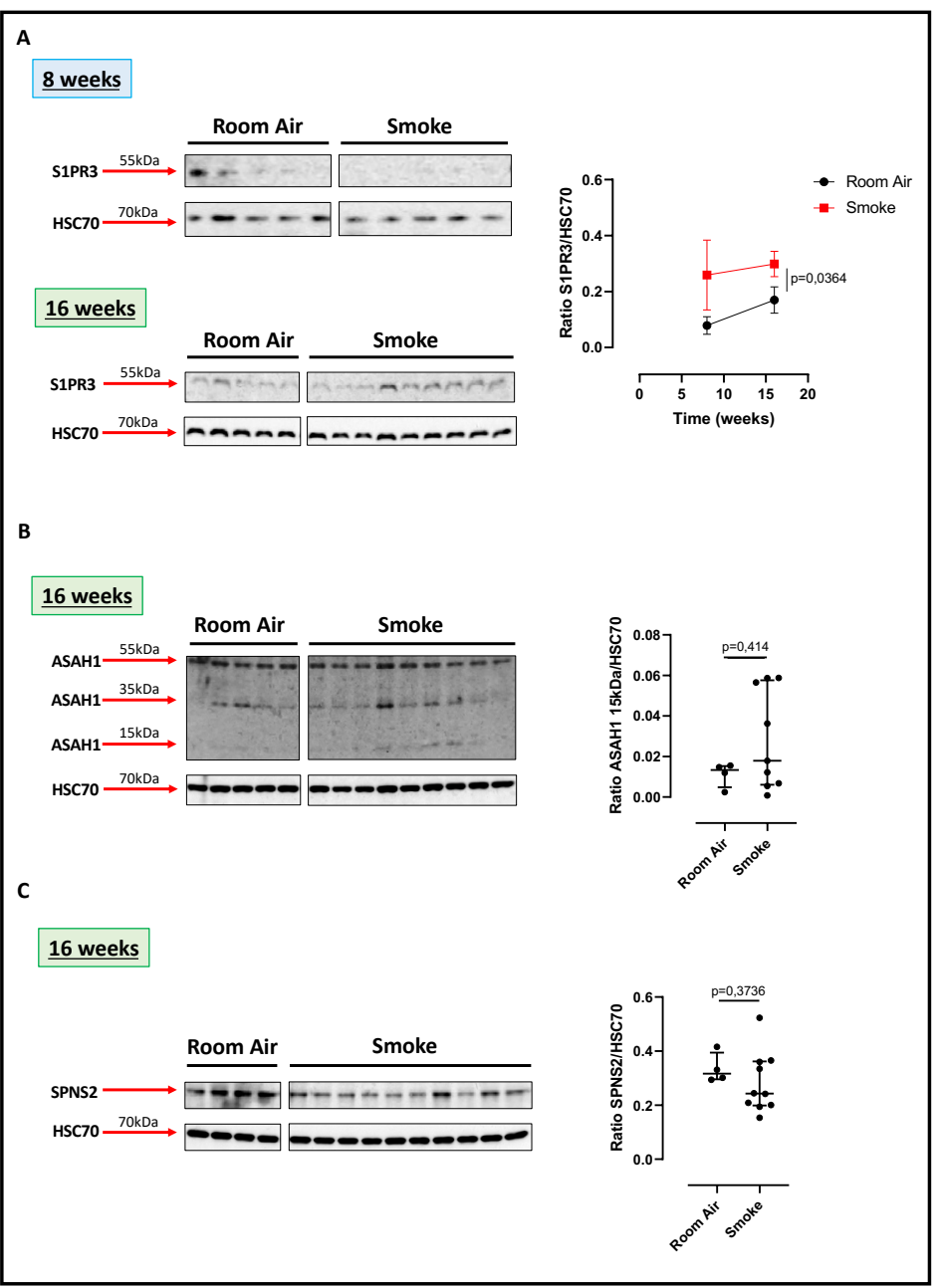

alveolar structure, collagen deposition, inducing an emphysematous pattern typical of chronic obstructive pulmonary disease (COPD) [21]. In these experimental conditions, S1PR3 was higher expressed in the lung of smoking mice at 16 weeks compared to 8 weeks and to the relative control group (Room Air) (Fig. 2A). Again, we were not able to detect S1PR1 (data not shown).

To evaluate whether sphingosine was produced starting from the metabolism of the ceramide, we first analyzed the expression of the ceramidase (ASAH1). The precursor form of the acid ceramidase (55kDa) was similarly expressed in the lung of Room Air and smoking group of mice exposed for 16 weeks (Fig. 2B). Instead, the smaller proteic fragments (35 and $15 \mathrm{kDa}$ ), representing the active form of the enzyme, seemed (not in a statistical manner) to be higher in the lung of smoking mice than in the Room Air group (Fig. 2B).

Interestingly, the expression of SPNS2 was higher in the lung of the Room Air group than in mice exposed for 16 weeks to smoke (Fig. 2C).

Therefore, the presence of smaller fragments of the ceramidase, 15 and $35 \mathrm{kDa}$, may imply the formation of S1P following smoking exposure. However, this effect was not correlated to the expression of SPNS2, S1P inside-out transporter, suggesting that smokeinduced S1P holds an intracellular signal rather than an extracellular/autocrine/paracrine signal. 


\section{S1P pathway in a mouse model of carcinogen-induced lung cancer}

Benzo(a)pyrene $(\mathrm{BaP})$ is one of the major components of tobacco smoking that has carcinogen-like activities. Therefore, in order to understand the role of the sphingosine/S1P axis in a tobacco-induced lung cancer, we used BaP that was instilled into $\mathrm{C} 57 \mathrm{Bl} / 6$ mice intratracheally, according to the experimental protocol described in the Material and Method section. The administration of $\mathrm{BaP}$ induced the formation of tumor lesions into the lung of mice compared to the vehicle group (acetone 0.1\%) (Fig. 3A and 3B). The lung of BaP-treated mice was characterized by the presence of immunosuppressive cells such as myeloid-derived suppressor cells (MDSCs: identified as CD $11 \mathrm{~b}^{+} \mathrm{Gr}-1^{+}$Arginase- $1^{+}$) (Fig. 3C) and T regulatory cells (Treg: identified as $\mathrm{CD}^{+} \mathrm{CD} 4^{+} \mathrm{CD} 25^{+} \mathrm{FoxP} 3^{+}$) (Fig. 3D). Both the percentage of MDSCs (Fig. 3C) and Treg (Fig. 3D) were statically higher in BaP-treated mice than vehicle mice.

The expression of S1PR1 at 16 weeks was significantly higher in the lung of BaP-treated mice (Fig. 3E). Similarly, the expression of S1PR3 at 16 weeks was higher in the carcinogeninduced lung tumor model than the vehicle group (Fig. 3F). Because the vehicle group was treated with acetone $0.1 \%$, we also analyzed naive (non-treated mice) as comparison (Fig. $3 \mathrm{E}$ and $3 \mathrm{~F}$ ).

To prove that sphingosine was phosphorylated, we used lung homogenates of Room Air, smoking- and BaP-treated mice and performed LC-MS/MS experiments. We found that the lung of Room Air mice had the highest content of sphingosine (Fig. 4A), which did not differ in terms of Peak Area than S1P, implying a physiological role of sphingosine/S1P in the lung, as already reported [3]. Instead, the lung of smoking and BaP-treated mice had lower levels of sphingosine (Fig. 4A), which corresponded to lower levels of S1P in the sole smoking mice but not in BaP-treated mice (Fig. 4B), implying that smoking induces the degradation of S1P and most likely its cellular activity is exerted, as already reported [23]. In support, the lung of smoking mice had higher active ceramidase (35 and $15 \mathrm{kDa}$, Fig. 2B) than Room Air mice, which leads to suggest that sphingosine is metabolized by ceramidase and then phosphorylated to S1P, active metabolite that interacts with its receptors [10].

\section{The intracellular S1P pathway leads to lung tumor cell proliferation}

In order to evaluate the molecular mechanism that binds sphingosine/S1P axis to lung carcinogenesis, we used a much simpler experimental approach by usinglung adenocarcinoma cells, A549. In order to evaluate whether S1P was able to induce tumor cell proliferation, we treated A549 cells with exogenous S1P. Surprisingly, we found that the stimulation of A549 cells with exogenous S1P (10 nM) was not able to induce A549 cells to proliferate (Fig. 5A). Therefore, we used CpG, a TLR9-ligand, that we previously demonstrated as involved in the release of endogenous S1P [18]. The stimulation of cells with CpG induced A549 cells to proliferate (Fig. 5B). To prove the role of S1P in this context and according to the insideout signaling of S1P, we blocked S1PR3, with a potent and selective receptor antagonist (S1PR3a). Interestingly and in support to what observed with the exogenous S1P (Fig. 5A), the pharmacological blockade of the extracellular S1PR3 did not alter A549 cell proliferation after CpG treatment (Fig. 5B). Similarly, the blockade of SPNS2 did not statistically modify the CpG-increased percentage of $\mathrm{CFSE}^{+}$cells (Fig. 5C). In sharp contrast, the inhibition of SPHK I/II, by means of SKI II inhibitor, responsible for sphingosine phosphorylation, completely reduced A549 proliferation after CpG treatment (Fig. 5D). Therefore, our next hypothesis was to understand the intracellular activity of the endogenous S1P.

Interestingly, we found that S1PR3 was also expressed into the nucleus of A549 cells (Fig. 5E). It has to be pointed out that the treatment of cells with CpG increased S1PR3 expression into the nucleus compared to the cytosol/membrane compartment (Fig. 5E). Importantly, we found that SPHK II was present into the nucleus and not in the cytosol/ membrane compartments (Fig. 5E), supporting the hypothesis that the pro-tumor activity of $\mathrm{S} 1 \mathrm{P}$ is intracellular and more specifically is related to the presence of S1PR3 into the nucleus.

To further prove our hypothesis, we analyzed the expression of S1PR3 in Room Air, smoking and BaP-treated mice by means of fluorescence staining to correlate the in vivo data (Fig. 1, 2 and 3) to the in vitro results (Fig. 5). As hypothesized, S1PR3 was overexpressed 
A

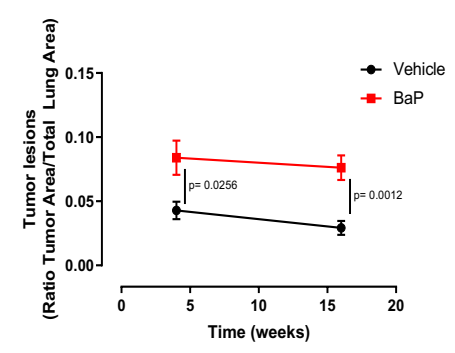

$\underline{4 \text { weeks }}$

C
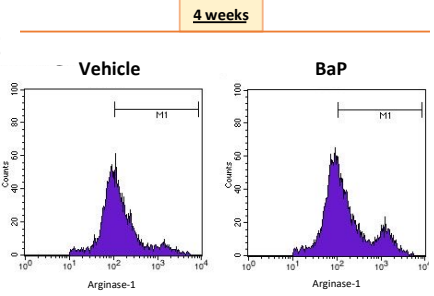

4 weeks
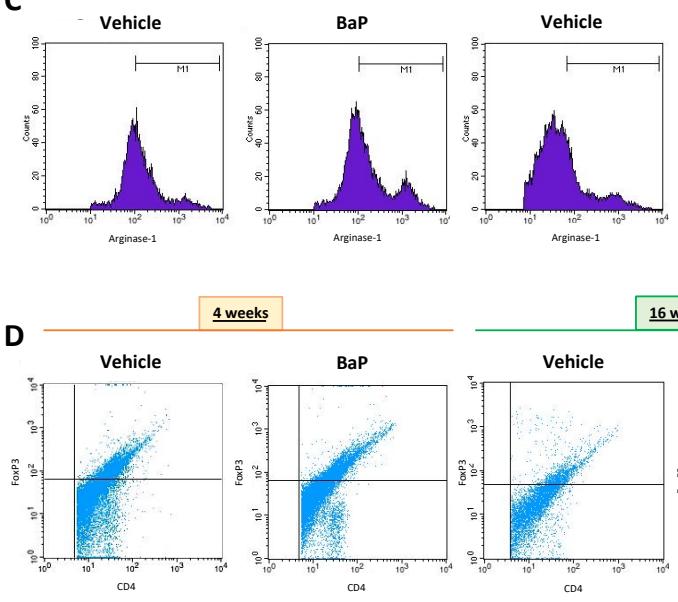

16 weeks
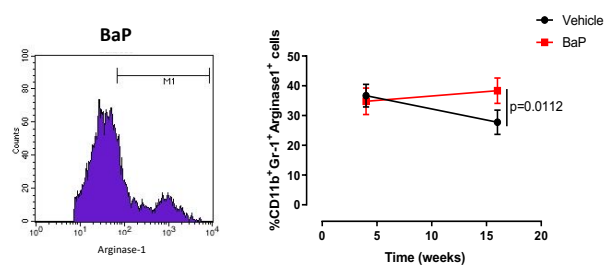

E
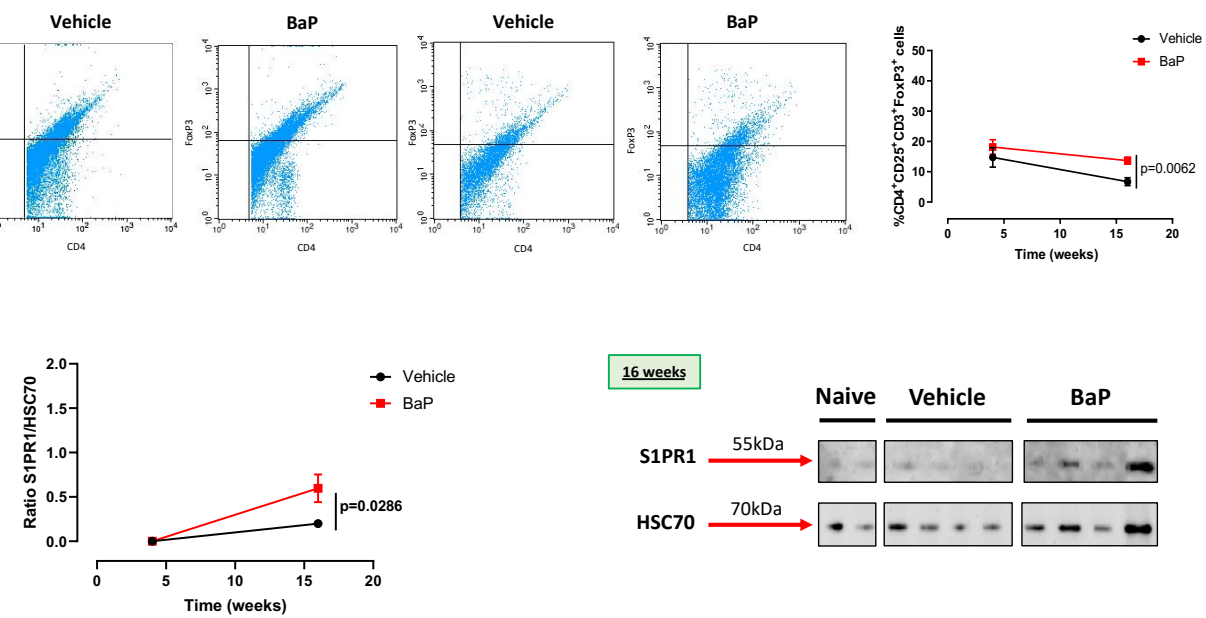

$\mathbf{F}$
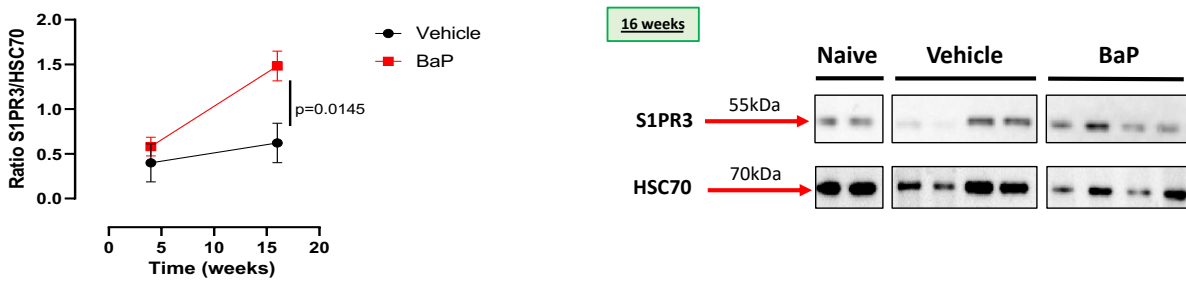

Fig. 3. S1P-dependent pathway in tumor bearing BaP-exposed mice. BaP-exposed mice (1 $\mu \mathrm{g} / \mathrm{mouse})$ developed lung tumor lesion compared to vehicles ( $0.1 \%$ acetone) both after 4 and 16 weeks of treatment (A), as shown in the H\&E picture in panel B, representative pictures of Vehicle- and BaP-exposed mice at 16 weeks. Flow cytometry analysis show that the recruitment of MDSCs (identified as CD11 $\mathrm{b}^{+} \mathrm{Gr}-1^{+}$Arginase $1^{+}$) (C) and Treg (identified as $\mathrm{CD} 4{ }^{+} \mathrm{CD} 3^{+} \mathrm{CD} 25^{+} \mathrm{FoxP}^{+}$) (D) is increased in the lung of BaP-treated mice compared to vehicle after 16 weeks of treatment. Histograms represent the percentage of Arginase- $1^{+}$MDSCs on vehicles- vs BaP-exposed mice at 4 and 16 weeks of treatment; dot plots show the Treg percentage recruited in the lungs of vehicles- vs BaP-exposed mice at 4 and 16 weeks of treatment. S1PR1 (55kDa) (E) and S1PR3 (55kDa) (F) lung expression is significantly increased after 16 weeks of exposure to carcinogen; the blots represent the different expression of S1PR1 and S1PR3 in the lung of BaP-exposed mice compared to the vehicles, after 16 weeks of treatment. HSC70 was used as loading control. The quantitative analysis was performed by ImageJ software. Data represents median \pm interquartile range. Statistical differences were performed by using Mann-Whitney t test. Vehicle: 0,1\% acetone; BaP: Benzo(a)pyrene. 


\section{Cellular Physiology Cell Physiol Biochem 2021;55:539-552 \begin{tabular}{ll|l} 
and Biochemistry & $\begin{array}{l}\text { DOl: 10.33594/000000431 } \\
\text { Published online: } 3 \text { September } 2021\end{array}$ & $\begin{array}{l}\text { O 2021 The Author(s). Published by } \\
\text { Cell Physiol Biochem Press GmbH\&Co. KG }\end{array}$ \\
\cline { 2 - 3 }
\end{tabular} \\ Terlizzi et al.: Intracellular Activity of S1P in Lung Tumor Cells}

Fig. 4. LC-MS/MS experiments on lung homogenates of Room Air, smoking- and BaP-exposed mice. LC-MS/MS experiment shows that the levels of sphingosine (expressed as sphingosine peak area) are reduced in the lung smoking- and BaP-exposed mice, compared to Room Air group (A), while S1P (expressed as S1P peak area) is reduced only in the smoking group (B). Data are presented as dot plot, showing the median \pm interquartile range. Statistical differences were assessed with Mann-Whitney t test. S1P: sphingosine-1-phosphate; BaP: Benzo(a)pyrene.

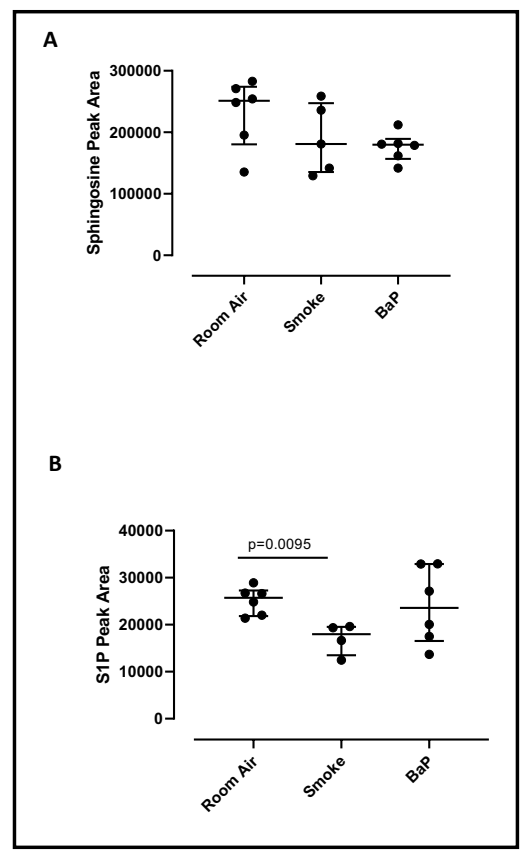

in the lung of BaP-treated mice compared to Room Air and smoking groups and it showed both membrane and nuclear localization (Fig. 6A, white arrows). Similarly, human lung cancer tissues expressed both membrane and nuclear S1PR3 compared to non-cancerous tissues (Fig. 6B, white arrows). These data confirm our hypothesis on the role of S1P in lung carcinogenesis via the activation of the nuclear S1PR3.

\section{Discussion}

High levels of S1P are associated to tumor progression $[24,25]$. In this study we highlighted a molecular mechanism underlying S1P-mediated tumor cell proliferation by using samples from NSCLC patients and smoke-exposed and BaP-induced lung tumor-bearing mice. We found that the common matrix to the three experimental conditions underlies the expression of S1PR3. In depth, the nuclear expression of S1PR3 was associated to lung cancer proliferation. Indeed, the blockade of the membrane S1PR3 did not alter tumor cell proliferation as, instead, in the case of CpG-treated cells where SPHK I/II were inhibited.

In our previous study we found that the activation of TLR-9, but not of TLR-4, was involved in the generation of S1P by means of ceramidase and SPHK I/II activation in tumor cells but not in non-cancerous cells. Through an inside-out signaling, newly formed S1P supported TLR-9/NF- $\kappa B-m e d i a t e d$ TNF- $\alpha$ release mainly via S1PR3 [18]. Here, instead, we found that the intracellular S1PR3 was related to tumor cell proliferation, being overexpressed in the nuclei of A549 cells, smoking mice, tumor bearing BaP-treated mice and human lung cancer tissues. The pharmacological inhibition of the membrane S1PR3, did not alter CpG-induced tumor cell proliferation. In this context, we used CpG to evaluate cell proliferation because the exogenous S1P was not able to induce proliferation, further supporting our hypothesis on the intracellular signaling of the endogenous S1P. Moreover, the choice to use CpG derived from our previous study in that the stimulation of TLR-9 led to the generation of endogenous S1P [18], which can either be transported in an inside-out mode by means of SPNS2, or it can lead to the activation of S1PR3 into the nucleus. The limitation of this study is a direct evidence that the sole nuclear S1PR3 is involved in proliferation signaling, mainly because of technical limitation, but both murine and human samples supported our hypothesis about the role of S1PR3 in lung carcinogenesis. In support, Spiegel and collaborators mentioned 


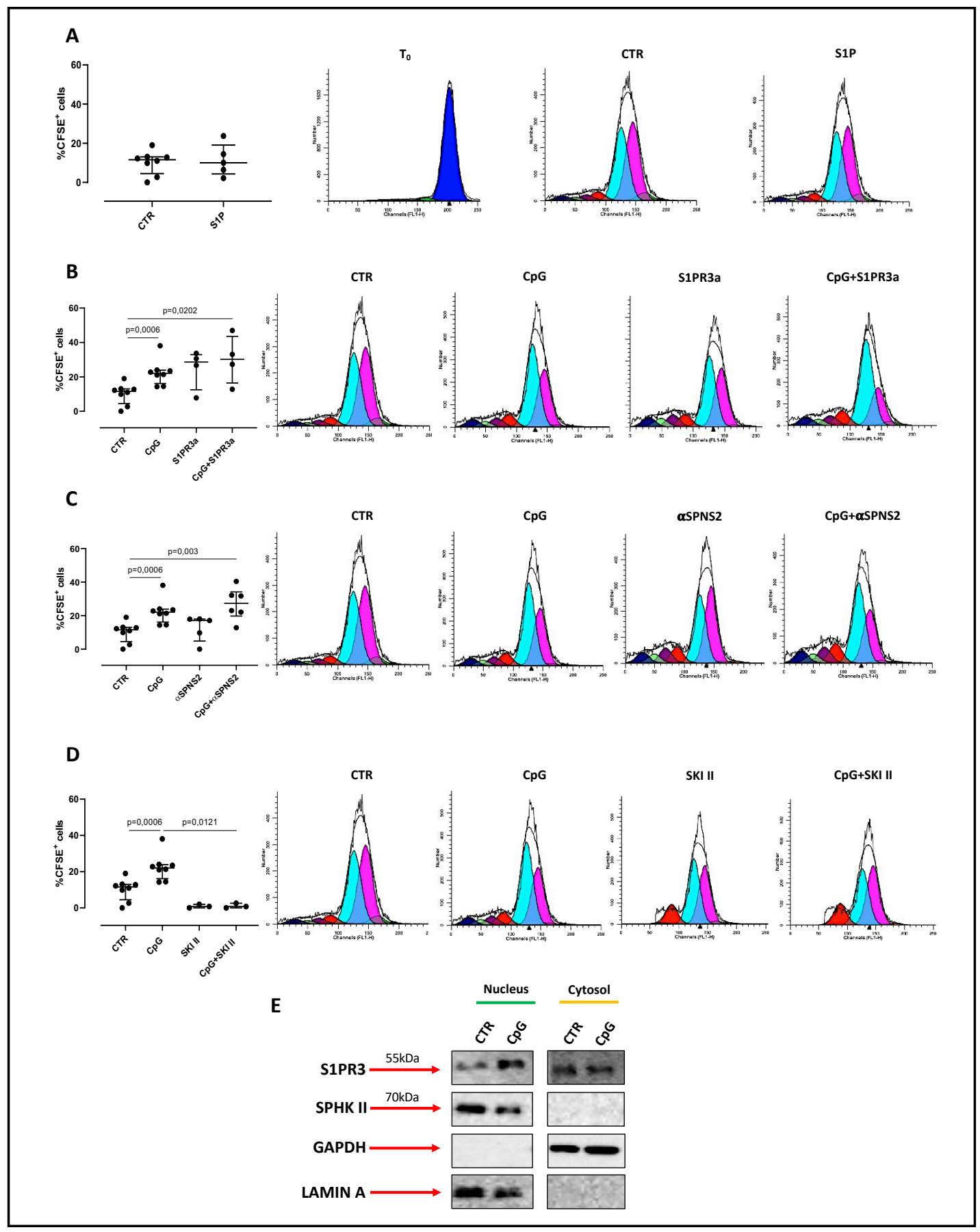

Fig. 5. Lung tumor cells proliferation is induced by S1P-dependent intracellular signaling. Proliferation experiment shows that S1P (10 nM) does not induce proliferation of A549 cells (A). The blocked of S1PR3 (B) and of SPNS2 (C) does not alter the proliferation induced by CpG. The inhibition of SPHK I/II reduces the A549 proliferation CpG-induced (D). G4 (violet peak) and G5 (light blue peak) generation of A549 $\mathrm{CFSE}^{+}$(FL1-H) were considered. E) S1PR3 is expressed in both nuclear and cytosolic compartment of A549 cells, while SPHK II is only expressed in the nucleus. GAPDH was used as cytosolic loading protein and LAMIN A as nuclear loading protein. Data are presented as dot plot, showing the median \pm interquartile range. Statistical differences were assessed with Ordinary ONE-Way ANOVA followed by Tukey's multiple comparison test. S1P: sphingosine-1-phosphate, 10nM; CpG: unmethylated Cytosine phosphate Guanosineoligodeoxynucleotides, $1 \mu \mathrm{g} / \mathrm{ml}$; S1PR3a: sphingosine-1-phosphate receptor 3 antagonist (TY52156), $10 \mu \mathrm{M}$; $\alpha$ SPNS2: anti-Spinster homolog 2 antibody, $10 \mathrm{ng} / \mathrm{ml}$; SKI II, selective inhibitor of sphingosine kinases, $10 \mu \mathrm{M}$; S1PR3: sphingosine-1-phosphate receptor 3; SPHK II: sphingosine kinase II. 


\section{Cellular Physiology \begin{tabular}{ll|l} 
and Biochemistry $10.33594 / 000000431$ & $\begin{array}{l}\text { Published online: } 3 \text { September } 2021 \\
\text { Pell } 21 \text { The Author(s). Published by } \\
\text { Cell Physiol Biochem Press GmbH\&Co. KG }\end{array}$ \\
\hline
\end{tabular}

Fig. 6. Immunofluorescence analyses for S1PR3 performed on mice left lung cryosections and on human non-cancerous vs lung cancer (LK) tissues. A) S1PR3 is overexpressed in the lung of BaP-treated mice compared to Room Air group and smoking mice at 16 weeks, and is preferentially localized in the nuclei (white arrows). B) Lung cancer patients show high levels of nuclear S1PR3 (white arrows) compared to noncancerous patients. Magnification: 40X; scale bar: $1000 \mu \mathrm{m}$. S1PR3: sphingosine1-phosphate receptor 3; BaP: Benzo(a) pyrene.

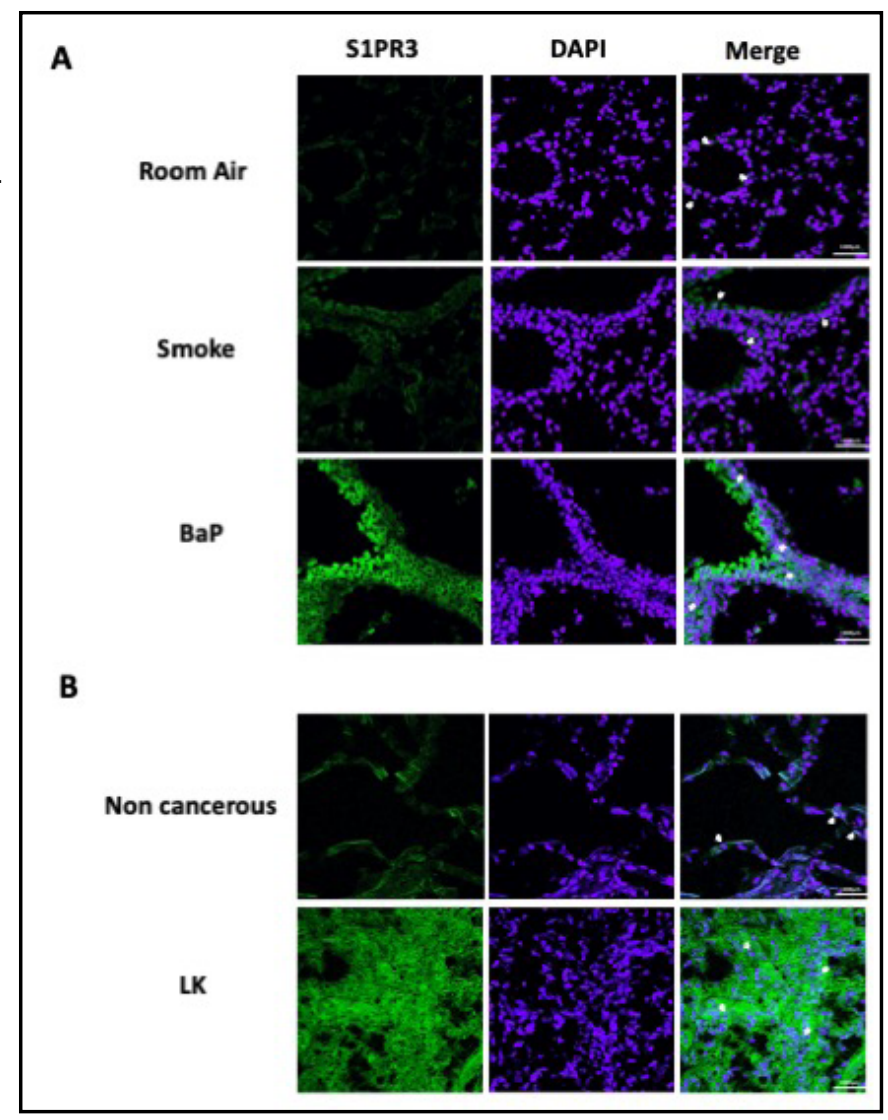

that besides S1P activity on its cell surface receptors, it can also have direct intracellular targets [26]. Several crucial studies have clearly shown that, S1P has specific intracellular actions that are independent of its cell surface receptors. For example, activation of Ras and ERK signaling pathways by VEGF requires SPHK I and is independent of S1P receptors [27], in contrast to cytoskeletal rearrangements and cell locomotion [28]. Similarly, TNF- $\alpha$ stimulates SPHK I, leading to the activation of the nuclear transcription factor NF- $\kappa \mathrm{B}$, which is essential for the prevention of apoptosis [29]. In our study, instead, we found that the nuclear S1PR3 is associated to the nuclear SPHK II and could be responsible for tumor growth. It still remains to elucidate the molecular mechanism by which this phenomenon could happen. However, it was already proved that S1P can interfere with the epigenetic context of tumor cells, or more simply it can be endogenously released according to the deregulated synthesizing mechanisms via other receptor triggering [30, 31]. In particular, because TLR-9 is overexpressed in lung cancer cells and in lung infiltrated immunesuppressive cells, responsible for tumor progression in vivo [32], it is plausible that the endogenous TLR-9-induced S1P can on one side favor pro-inflammatory mechanisms in the tumor microenvironment via the activation of cell surface receptors, but on the other tumor progression via the nuclear S1PR3/SPHK II axis.

Another important issue that is worthy to be highlighted in this study is the overexpression of S1PR3 in both smoke- and BaP-exposed mice, which reflect what happens in human samples of NSCLC, all of which were smokers. Similarly to what we found in in vitro studies [18], we observed that S1PR3/S1P signaling during smoking-induced lung inflammation is associated to immunosuppressive cells and to the presence of tumor lesions in both mice and humans. However, we have to point out that all lung (murine and human) samples, showed in Fig. 1-3, were lung homogenates which did not discriminate the expression of S1PR3 between cell surface and nucleus. Though, immunofluorescent analyses showed S1PR3 nuclear overexpression, particularly in tumor bearing BaP-treated mice and in human lung cancer tissues. 


\section{Conclusion}

In conclusion, our findings further puzzle up the pathological role of S1P which on one side can exacerbate the inflammatory pulmonary milieu via the cell surface S1PR3, and on the other it is involved in lung tumor proliferation. Although the limitations of this study, we were able to prove that the nuclear S1PR3/SPHK II axis is involved in lung tumor cell proliferation, which is a novel molecular mechanism which could provide differential therapeutic approaches especially in non-responsive lung cancer patients.

\section{Acknowledgements}

\section{Author Contributions}

MT and CC designed and performed experiments; GF and MCM performed LC-MS/MS data; IDR, PS, RS (Salvi) recruited NSCLC patients; MT, AP and RS (Sorrentino) statistically analyzed and interpreted data and wrote the manuscript.

\section{Funding}

This study was supported by FARB-2017-2020 and Italian Association against Leukemia (AIL) in favor of RS.

\section{Statement of Ethics}

The authors have no ethical conflicts to disclosure. This study was carried out in strict accordance with the recommendations in the Guide for Care and Use of Laboratory Animals of the Health National Institute, and was approved by the institutional review board and by the Ethical Committee.

\section{Disclosure Statement}

The authors have no conflicts of interest to declare.

\section{References}

1 Pyne S, Adams DR, Pyne NJ: Sphingosine 1-phosphate and sphingosine kinases in health and disease: Recent advances. Prog Lipid Res 2016;62:93-106.

2 Roviezzo F, Sorrentino R, Bertolino A, De Gruttola L, Terlizzi M, Pinto A, Napolitano M, Castello G, D’Agostino B, Ianaro A, Sorrentino R, Cirino G: S1P-induced airway smooth muscle hyperresponsiveness and lung inflammation in vivo: molecular and cellular mechanisms. Br J Pharmacol 2015;172:1882-1893.

3 Hannun YA, Obeid LM: Sphingolipids and their metabolism in physiology and disease. Nat Rev Mol Cell Biol 2018;19:175-191.

4 Riboni L, Abdel Hadi L, Navone SE, Guarnaccia L, Campanella R, Marfia G: Sphingosine-1-Phosphate in the Tumor Microenvironment: A Signaling Hub Regulating Cancer Hallmarks. Cells 2020;9:337.

5 Proia RL, Hla T: Emerging biology of sphingosine-1-phosphate: its role in pathogenesis and therapy. J Clin Invest 2015;125:1379-1387.

6 Gault CR, Obeid LM, Hannun YA: An overview of sphingolipid metabolism: from synthesis to breakdown. Adv Exp Med Biol 2010;688:1-23.

7 Spiegel S, Maczis MA, Maceyka M, Milstien S: New insights into functions of the sphingosine-1-phosphate transporter SPNS2. J Lipid Res 2019;60:484-489.

8 Sorrentino R, Bertolino A, Terlizzi M, Iacono VM, Maiolino P, Cirino G, Roviezzo F, Pinto A: B cell depletion increases sphingosine-1-phosphate-dependent airway inflammation in mice. Am J Respir Cell Mol Biol 2015;52:571-583. 
9 Roviezzo F, Sorrentino R, Iacono VM, Brancaleone V, Terlizzi M, Riemma MA, Bertolino A, Rossi A, Matteis M, Spaziano G, Pinto A, D’Agostino B, Cirino G: Disodium cromoglycate inhibits asthma-like features induced by sphingosine-1-phosphate. Pharmacol Res 2016;113:626-635.

10 Brinkmann V: Sphingosine 1-phosphate receptors in health and disease: mechanistic insights from gene deletion studies and reverse pharmacology. Pharmacol Ther 2007;115:84-105.

11 Cartier A, Leigh T, Liu CH, Hla T: Endothelial sphingosine 1-phosphate receptors promote vascular normalization and antitumor therapy. Proc Natl Acad Sci U S A 2020;117:3157-3166.

12 Balaji Ragunathrao VA, Anwar M, Akhter MZ, Chavez A, Mao Y, Natarajan V, Lakshmikanthan S, Chrzanowska-Wodnicka M, Dudek AZ, Claesson-Welsh L, Kitajewski JK, Wary KK, Malik AB, Mehta D: Sphingosine-1-Phosphate Receptor 1 Activity Promotes Tumor Growth by Amplifying VEGF-VEGFR2 Angiogenic Signaling. Cell Rep 2019;29:3472-3487.e4.

13 Rostami N, Nikkhoo A, Ajjoolabady A, Azizi G, Hojjat-Farsangi M, Ghalamfarsa G, Yousefi B, Yousefi M, Jadidi-Niaragh F: S1PR1 as a Novel Promising Therapeutic Target in Cancer Therapy. Mol Diagn Ther 2019;23:467-487.

14 Lee HM, Lo KW, Wei W, Tsao SW, Chung GTY, Ibrahim MH, Dawson CW, Murray PG, Paterson IC, Yap LF: Oncogenic S1P signalling in EBV-associated nasopharyngeal carcinoma activates AKT and promotes cell migration through S1P receptor 3. J Pathol 2017;242:62-72.

15 Zhao J, Liu J, Lee JF, Zhang W, Kandouz M, VanHecke GC, Chen S, Ahn YH, Lonardo F, Lee MJ: TGF- $\beta$ /SMAD3 Pathway Stimulates Sphingosine-1 Phosphate Receptor 3 Expression: IMPLICATION OF SPHINGOSINE-1 PHOSPHATE RECEPTOR 3 IN LUNG ADENOCARCINOMA PROGRESSION. J Biol Chem 2016;291:2734327353.

16 Rivera J, Proia RL, Olivera A: The alliance of sphingosine-1-phosphate and its receptors in immunity. Nat Rev Immunol 2008;8:753-763.

17 Olesch C, Sirait-Fischer E, Berkefeld M, Fink AF, Susen RM, Ritter B, Michels BE, Steinhilber D, Greten FR, Savai R, Takeda K, Brüne B, Weigert A: S1PR4 ablation reduces tumor growth and improves chemotherapy via CD8+ T cell expansion. J Clin Invest 2020;130:5461-5476.

18 Terlizzi M, Colarusso C, Ferraro G, Monti MC, Cerqua I, Roviezzo F, Pinto A, Sorrentino R: Sphingosine1-Phosphate Contributes to TLR9-Induced TNF- $\alpha$ Release in Lung Tumor Cells. Cell Physiol Biochem 2021;55:222-234.

19 Milara J, Navarro R, Juan G, Peiró T, Serrano A, Ramón M, Morcillo E, Cortijo J: Sphingosine-1-phosphate is increased in patients with idiopathic pulmonary fibrosis and mediates epithelial to mesenchymal transition. Thorax 2012;67:147-156.

20 Alberg AJ, Armeson K, Pierce JS, Bielawski J, Bielawska A, Visvanathan K, Hill EG, Ogretmen B: Plasma sphingolipids and lung cancer: a population-based, nested case-control study. Cancer Epidemiol Biomarkers Prev 2013;22:1374-1382.

21 Colarusso C, Terlizzi M, Lamort AS, Cerqua I, Roviezzo F, Stathopoulos G, Pinto A, Sorrentino R: Caspase-11 and AIM2 inflammasome are involved in smoking-induced COPD and lung adenocarcinoma. Oncotarget 2021;12:1057-1071.

22 Bielawski J, Szulc ZM, Hannun YA, Bielawska A: Simultaneous quantitative analysis of bioactive sphingolipids by high-performance liquid chromatography-tandem mass spectrometry. Methods 2006;39:82-91.

23 De Cunto G, Brancaleone V, Riemma MA, Cerqua I, Vellecco V, Spaziano G, Cavarra E, Bartalesi B, D’Agostino B, Lungarella G, Cirino G, Lucattelli M, Roviezzo F: Functional contribution of sphingosine-1-phosphate to airway pathology in cigarette smoke-exposed mice. Br J Pharmacol 2020;177:267-281.

24 Hait NC, Maiti A: The Role of Sphingosine-1-Phosphate and Ceramide-1-Phosphate in Inflammation and Cancer. Mediators Inflamm 2017;2017:4806541.

25 Wang P, Yuan Y, Lin W, Zhong H, Xu K, Qi X: Roles of sphingosine-1-phosphate signaling in cancer. Cancer Cell Int 2019;19:295.

26 Maceyka M, Harikumar KB, Milstien S, Spiegel S: Sphingosine-1-phosphate signaling and its role in disease. Trends Cell Biol 2012;22:50-60.

27 Shu X, Wu W, Mosteller RD, Broek D: Sphingosine kinase mediates vascular endothelial growth factorinduced activation of ras and mitogen-activated protein kinases. Mol Cell Biol 2002;22:7758-7768. 


\section{Cellular Physiology Cell Physiol Biochem 2021;55:539-552}

\begin{tabular}{ll|l} 
and Biochemistry & $\begin{array}{l}\text { DOl: 10.33594/000000431 } \\
\text { Published online: } 3 \text { September } 2021\end{array}$ & $\begin{array}{l}\text { O 2021 The Author(s). Published by } \\
\text { Cell Physiol Biochem Press GmbH\&Co. KG }\end{array}$ \\
\cline { 2 - 3 }
\end{tabular}

Terlizzi et al.: Intracellular Activity of S1P in Lung Tumor Cells

28 Olivera A, Rosenfeldt HM, Bektas M, Wang F, Ishii I, Chun J, Milstien S, Spiegel S: Sphingosine kinase type 1 induces G12/13-mediated stress fiber formation, yet promotes growth and survival independent of G protein-coupled receptors. J Biol Chem 2003;278:46452-46460.

29 Xia P, Wang L, Moretti PA, Albanese N, Chai F, Pitson SM, D’Andrea RJ, Gamble JR, Vadas MA: Sphingosine kinase interacts with TRAF2 and dissects tumor necrosis factor-alpha signaling. J Biol Chem 2002;277:7996-8003.

30 Wang W, Toran PT, Sabol R, Brown TJ, Barth BM: Epigenetics and Sphingolipid Metabolism in Health and Disease. Int J Biopharm Sci 2018;1:105.

31 di Villa Bianca Rd, Sorrentino R, Sorrentino R, Imbimbo C, Palmieri A, Fusco F, Maggi M, De Palma R, Cirino G, Mirone V: Sphingosine 1-phosphate induces endothelial nitric-oxide synthase activation through phosphorylation in human corpus cavernosum. J Pharmacol Exp Ther 2006;316:703-708.

32 Sorrentino R, Morello S, Giordano MG, Arra C, Maiolino P, Adcock IM, Pinto A: CpG-ODN increases the release of VEGF in a mouse model of lung carcinoma. Int J Cancer 2011;128:2815-2822. 